\title{
Temporal Prediction Signals for Periodic Sensory Events in the Primate Central Thalamus
}

\author{
${ }^{\circledR}$ Kei Matsuyama and ${ }^{\circledR}$ Masaki Tanaka \\ Department of Physiology, Hokkaido University School of Medicine, Sapporo 060-8638, Japan
}

Prediction of periodic event timing is an important function for everyday activities, while the exact neural mechanism remains unclear. Previous studies in nonhuman primates have demonstrated that neurons in the cerebellar dentate nucleus and those in the caudate nucleus exhibit periodic firing modulation when the animals attempt to detect a single omission of isochronous repetitive audiovisual stimuli. To understand how these subcortical signals are sent and processed through the thalamocortical pathways, we examined single-neuron activities in the central thalamus of two macaque monkeys (one female and one male). We found that three types of neurons responded to each stimulus in the sequence in the absence of movements. Reactive-type neurons showed sensory adaptation and gradually waned the transient response to each stimulus. Predictive-type neurons steadily increased the magnitude of the suppressive response, similar to neurons previously reported in the cerebellum. Switch-type neurons initially showed a transient response, but after several cycles, the direction of firing modulation reversed and the activity decreased for each repetitive stimulus. The time course of Switch-type activity was well explained by the weighted sum of activities of the other types of neurons. Furthermore, for only Switch-type neurons the activity just before stimulus omission significantly correlated with behavioral latency, indicating that this type of neuron may carry a more advanced signal in the system detecting stimulus omission. These results suggest that the central thalamus may transmit integrated signals to the cerebral cortex for temporal information processing, which are necessary to accurately predict rhythmic event timing.

Key words: temporal prediction; periodicity; phase transition; motor thalamus; single neuron; monkey

\section{Significance Statement}

Several cortical and subcortical regions are involved in temporal information processing, and the thalamus will play a role in functionally linking them. The present study aimed to clarify how the paralaminar part of the thalamus transmits and modifies signals for temporal prediction of rhythmic events. Three types of thalamic neurons exhibited periodic activity when monkeys attempted to detect a single omission of isochronous repetitive stimuli. The activity of one type of neuron correlated with the behavioral latency and appeared to be generated by integrating the signals carried by the other types of neurons. Our results revealed the neuronal signals in the thalamus for temporal prediction of sensory events, providing a clue to elucidate information processing in the thalamocortical pathways.

\section{Introduction}

Prediction of periodic event timing in the range of hundreds of milliseconds is crucial for a variety of everyday activities such as rhythm perception and movement synchronization. The

\footnotetext{
Received Aug. 17, 2020; revised Dec. 7, 2020; accepted Jan. 3, 2021.

Author contributions: K.M. and M.T. designed research; K.M. performed research; K.M. analyzed data; M.T. wrote the paper.

This work was supported partly by the Ministry of Education, Culture, Sports, Science and Technology of Japan (Grants 17H03539, 18H04928, and 18H05523). K.M. received a scholarship from the Takeda Science Foundation. We thank T. Mori, A. Hironaka, and H. Miyaguchi for assistance with animal care and surgery; M. Suzuki for administrative help; M. Takei and M. Kusuzaki at the Research Institute for Electronic Science for manufacturing some equipment; and all laboratory members for comments and discussions.

The authors declare no competing financial interests.

Correspondence should be addressed to Masaki Tanaka at masaki@med.hokudai.ac.jp.

https://doi.org/10.1523/JNEUROSCI.2151-20.2021

Copyright $\odot 2021$ the authors
}

cerebellum and basal ganglia are known to play a role in temporal prediction (Allman and Meck, 2012; Merchant et al., 2013; Paton and Buonomano, 2018), and the signals from these subcortical structures are sent through the thalamus to the cortex, which has also been implicated in temporal information processing (Rao et al., 1997; Harrington et al., 2011; Fujioka et al., 2012). Neuronal signals in the dorsomedial frontal cortex have been shown to represent the internal model of temporal estimation (Egger et al., 2019), which may originate from the thalamus. Indeed, previous studies in nonhuman primates have shown that neurons in the ventrolateral (VL) thalamus exhibit activities representing subjective passage of time during the preparation of self-initiated movements (Tanaka, 2007a; Wang et al., 2018). However, it remains unknown how thalamic neurons contribute to the temporal prediction of periodic events in the absence of movements. 
Several lines of evidence suggest that the thalamus is not only involved in relaying a copy of subcortical signals, but also in integrating signals from multiple sources (Rovó et al., 2012; Groh et al., 2014; Ahissar and Oram, 2015; Yamawaki and Shepherd, 2015). Anatomically, the VL thalamus receives inputs from the cerebellum and basal ganglia, and reciprocally connects with the frontal cortex (Kakei et al., 2001; McFarland and Haber, 2002). The adjacent centrolateral (CL) nucleus collects broad signals from multiple sources in the cerebral cortex, cerebellum, and brainstem, and in turn sends axons to the frontoparietal cortices and the striatum (Van der Werf et al., 2002; Saalmann, 2014; Redinbaugh et al., 2020). These thalamic nuclei, together with the paralaminar part of the mediodorsal (MD) nucleus, comprise the "central thalamus," which might play an active role in information processing for various cognitive functions (Schlag-Rey and Schlag, 1989; Schlag and Schlag-Rey, 2002; Haber and Calzavara, 2009; Tanaka and Kunimatsu, 2011; Prevosto and Sommer, 2013). Lesions in this part of the thalamus have been shown to cause impairments in movement decisions (Newman and Mair, 2007), loss of behavioral flexibility (Kato et al., 2018), deterioration of working memory (Mitchell and DalrympleAlford, 2006; Mair and Hembrook, 2008), difficulty in suppressing impulsive behavior (Kunimatsu and Tanaka, 2010), a delay of self-initiated actions (van Donkelaar et al., 2000; Tanaka, 2006), and dysfunction of arousal regulation (Schiff, 2008). Furthermore, neuronal recordings in experimental animals have revealed relevant sensory, motor, and cognitive signals (Wyder et al., 2003, 2004; Tanaka, 2007b; Costello et al., 2016), whereas their role in information processing for rhythm has not been investigated.

The aim of the present study was to understand the role of the central thalamus in the temporal prediction of periodic events. We conducted single-neuron recordings in monkeys performing the missing oddball detection task, in which the animals made an eye movement in response to a single omission of isochronous repetitive stimuli (Ohmae et al., 2013; Uematsu et al., 2017; Kameda et al., 2019). To detect stimulus omission, the animals had to predict each stimulus timing while maintaining eye fixation until the occurrence of an unexpected stimulus omission. Our previous studies using this behavioral paradigm demonstrated that neurons in the cerebellar dentate nucleus and those in the striatum exhibited periodic firing modulation that was gradually enhanced as the repetition progressed (Ohmae et al., 2013; Kameda et al., 2019). Because different nuclei in the central thalamus receive direct inputs from the deep cerebellar nuclei and the output nodes of the basal ganglia (Alexander et al., 1986; Parent and Hazrati, 1995; Bostan and Strick, 2018), neurons in the central thalamus likely transmit signals from these subcortical structures to the cerebral cortex. Considering the possible role of the central thalamus in active information processing (Bosch-Bouju et al., 2013), these signals might be modified before they reach the cerebral cortex.

\section{Materials and Methods}

Animal preparation. Two Japanese monkeys (Macaca fuscata, one female and one male, monkeys $\mathrm{M}$ and $\mathrm{Z}, 6.0-8.0 \mathrm{~kg}$ ) were used. All animal care and experimental procedures were evaluated and approved in advance by the Hokkaido University Animal Care and Use Committee. Animals were prepared for chronic experiments using the procedures described previously (Tanaka, 2005). Briefly, in separate surgeries under general isoflurane anesthesia, a pair of plastic head holders was fixed to the skull and an eye coil was implanted for monitoring eye movements. Analgesics were administered during each surgery and a few days following the surgery. After full recovery from the surgery, the monkeys were trained in the behavioral tasks. Once the animals were well trained and they could perform the task correctly in $>80 \%$ of trials, a third surgery was undertaken to implant a recording cylinder for vertical electrode penetration aimed at the central thalamus. The location of the cylinder was verified by magnetic resonance (MR) imaging. Antibiotics were administered as needed.

Visual stimuli and behavioral tasks. Experiments were conducted in a darkened booth and were controlled with a commercial data acquisition system (TEMPO, Reflective Computing). Visual stimuli were presented on a 24 inch cathode ray tube monitor (GDM-FW900, Sony; refresh rate, $60 \mathrm{~Hz}$ ) located $38 \mathrm{~cm}$ from the eyes (visual angle, $64^{\circ} \times 44^{\circ}$ ). We used the missing oddball detection task (Ohmae et al., 2013). Shortly after the monkeys started fixation on a central spot $\left(0.5^{\circ}\right.$, red square), the saccade target $\left(0.5^{\circ}\right.$, gray square) was presented at $16^{\circ}$ left or right of the fixation point. During the maintenance of fixation, a brief ( $35 \mathrm{~ms})$ audiovisual stimulus $\left(2^{\circ}\right.$ white square contour enclosing the fixation point and a $1500 \mathrm{~Hz}$ tone) was also presented repeatedly at a fixed interstimulus interval (ISI) of 100, 200, 300, 400, or $600 \mathrm{~ms}$ (Fig. 1A). One stimulus in the series was omitted after a random $2000-4800$ ms period (3600$4800 \mathrm{~ms}$ for trials with a $600 \mathrm{~ms}$ ISI). In response to the stimulus omission (missing oddball), the monkeys were required to make an immediate saccade to the visible target. If the monkeys did not make a saccade within $800 \mathrm{~ms}$ from the stimulus omission, the trial was aborted. In addition to this basic configuration, two additional conditions were also presented. In the "nontarget" trial (see Fig. 6), the audiovisual stimulus was presented repeatedly at a fixed ISI of $400 \mathrm{~ms}$, but the saccade target never appeared. The monkeys were only required to maintain fixation throughout the trial that terminated after three stimulus repetitions following the omission. In the "explicit deviant" trial (see Fig. 7), a green fixation point, rather than a red fixation point, was presented, and the repetitive stimulus (400 ms ISI) suddenly altered its color and frequency ( $2^{\circ}$ red filled square and $667 \mathrm{~Hz}$ tone). In response to this stimulus deviation, the monkeys made an immediate saccade to the target. These trials were randomly intermingled with the missing oddball trials.

Physiologic procedures. Single-neuron recordings were conducted in three thalami of two monkeys. The location of the central thalamus was verified based on MR images taken before the experiments. The recording sites in one animal were reconstructed from histologic sections obtained after the experiments (see below). Neuronal activity was recorded using single tungsten microelectrodes (FHC). The electrode was inserted vertically into the thalamus with the aid of a grid system (Crist Instrument) and a micromanipulator (MO-97S, Narishige). The neuronal signals were amplified, filtered $(300 \mathrm{~Hz}$ to $10 \mathrm{kHz})$, and monitored online using oscilloscopes and audio devices. Typically, the dorsal surface of the thalamus could be identified below the active callosal fibers and the lateral ventricle. Once a task-related neuron was encountered, the waveform of action potentials was isolated using the software with real-time template-matching algorithms (MSD, Alpha Omega Engineering).

Data acquisition and analysis. Eye movement data were directly obtained from the eye coil device (MEL-25, Enzanshi Kogyo). The data were digitized, sampled at $1 \mathrm{kHz}$, and saved in files during the experiments. The times of action potentials and other codes of task events were also stored in the same files for offline analyses that were performed using MATLAB (MathWorks). Data were included for the analysis when monkeys responded correctly within $800 \mathrm{~ms}$ from the occurrence of the oddball (i.e., omission or color deviation of the repetitive stimulus). We used the data of neurons recorded for eight or more blocks of trials for further quantitative analyses (mean $\pm \mathrm{SD}, 21.6 \pm 8.3$ trials ranged from $8-53$ trials $n=104$ neurons). For each neuron, the time course of the firing rate in multiple trials in each condition was obtained by computing the spike density that convolved the millisecond-by-millisecond occurrence of action potentials with a Gaussian kernel $(\sigma=$ $30 \mathrm{~ms}$ ). Unless otherwise noted, the quantitative measures of neuronal activity were based on the modulation of spike density during specific intervals during the task. Details of the quantitative measures are reported in the relevant text. Statistical significance was evaluated using paired or unpaired $t$ test, Kolmogorov-Smirnov test, Wilcoxon rank- 

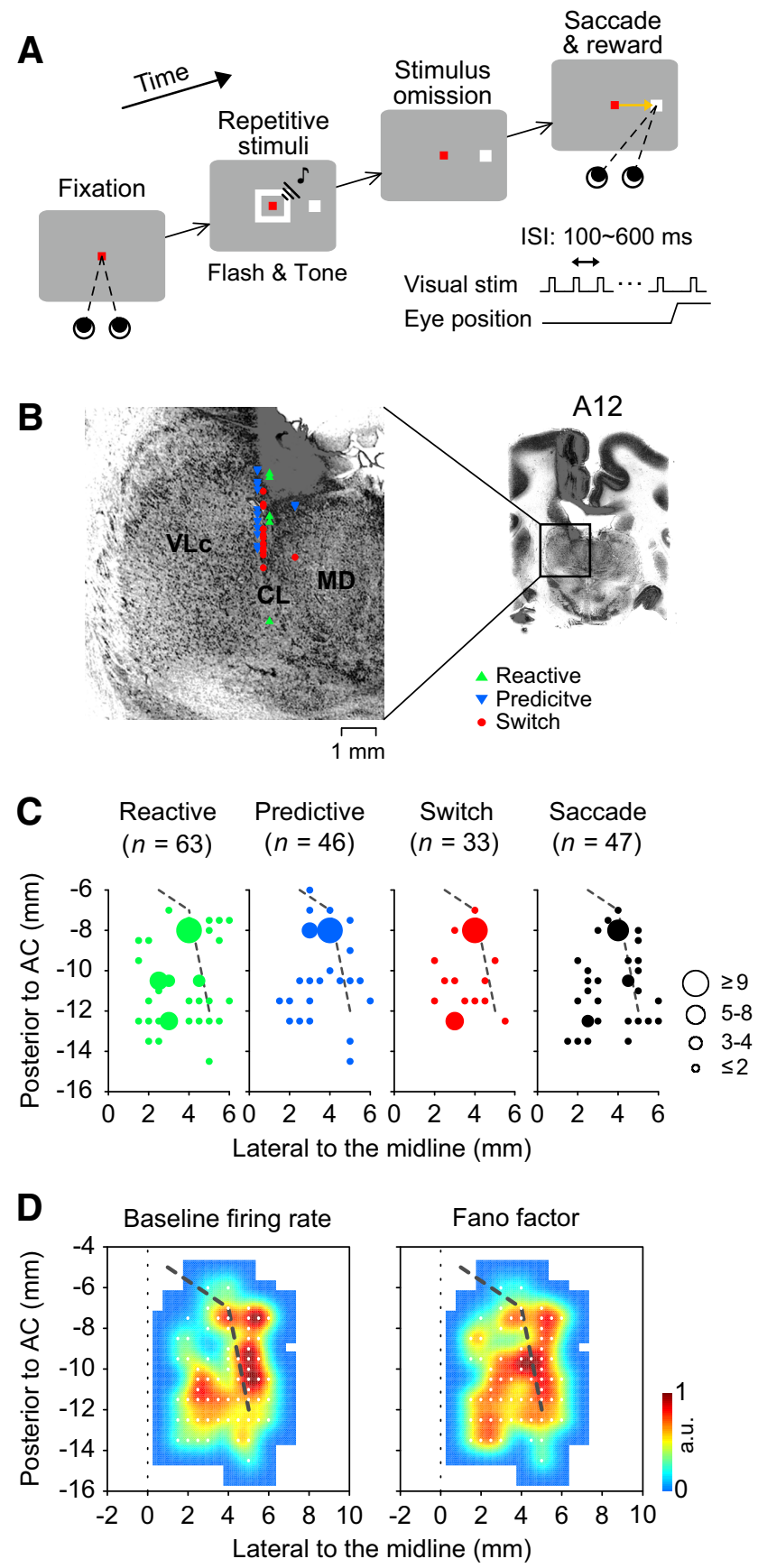

Figure 1. Behavioral paradigm and recording sites. $A$, Sequence of events in the missing oddball task. While animals gazed at the central fixation point (red square), a brief audiovisual stimulus (stim) was presented repeatedly at a fixed ISI that varied from trial to trial. In response to a single omission of the repetitive stimuli, monkeys made a saccade to the target located $16^{\circ}$ left or right of the fixation point. $\boldsymbol{B}$, Recording sites of different types of taskrelated neurons are plotted on the histologic section of monkey M. Because many neurons were recorded from a single coordinate of electrode penetration (A12, L4), data for Reactivetype (green triangle) and Predictive-type (blue) neurons are shifted horizontally for the purpose of presentation only. The historical section was $8 \mathrm{~mm}$ posterior to the $A C$ and $12 \mathrm{~mm}$ anterior to the interaural line. VLC, Caudal part of the VL nucleus. C, Dorsal view of the recording sites. Locations of electrode tracks are plotted according to the distance from the midline and AC. The size of circles indicates the number of recorded neurons at each coordinate. Black dashed line indicates the approximate location of the intralaminar thalamus. $\boldsymbol{D}$, Heatmaps represent the mean firing rate (left) and Fano factor (right) measured during the initial fixation period before the target onset. Data for each penetration (white dot) were averaged and filtered with two-dimensional Gaussians $(\sigma=0.5 \mathrm{~mm})$.

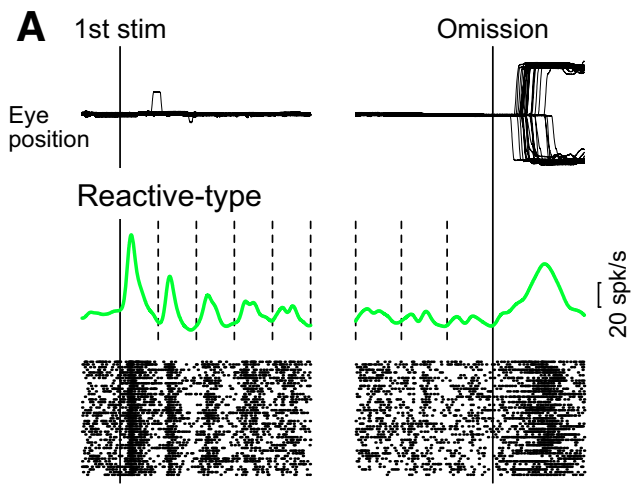

B Predictive-type

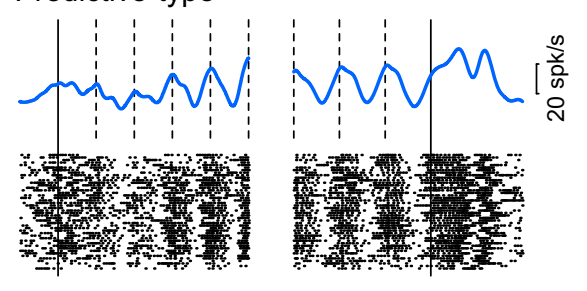

C Switch-type

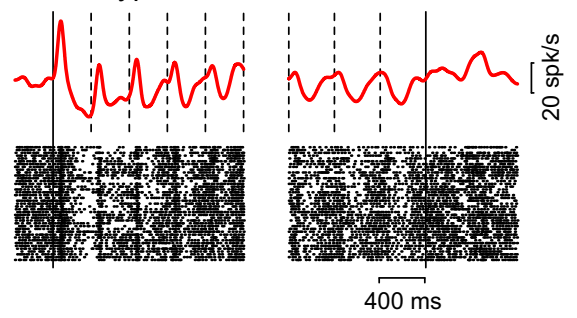

Figure 2. Representative examples of three types of thalamic neurons responding to each repetitive stimulus. $\boldsymbol{A}$, A Reactive-type neuron. Data are aligned with the first stimulus onset (left) or the stimulus omission (right). Each vertical dashed line represents stimulus timing that was separated by $400 \mathrm{~ms}$. B, A Predictive-type neuron. C, A Switch-type neuron. Note that this neuron initially showed facilitatory response to each stimulus, but the direction of firing modulation reversed as the repetition progressed. The timing of peak activity eventually synchronized with the stimulus onset (right). spk, Spike.

sum test, one-way ANOVA, or $\chi^{2}$ test. As circular statistics, WatsonWilliams test was also performed with the CircStat toolbox for MATLAB (Berens, 2009).

Classification of neurons. Thalamic neurons responding to the repetitive stimuli were classified into three groups according to the direction of firing modulation for each stimulus in trials with a $400 \mathrm{~ms}$ ISI (Figs. 2, $3)$. Because some neurons reversed the direction of firing modulation during each trial, neuronal activity was evaluated separately for the first three ISIs (between the first and the fourth stimuli) and the last three ISIs (ending at the stimulus omission). For both early and late intervals, the spike densities were averaged across the three ISIs to obtain the data of the $400 \mathrm{~ms}$ ISI. The response direction (facilitatory or suppressive) was determined based on the direction of maximal firing modulation relative to the activity at the time of stimulus onset. To assess the magnitude of firing modulation, we initially searched for the maximal response (peak or trough in spike density) during the $100-350 \mathrm{~ms}$ following the stimulus onset and then measured the actual spike count during the 50 $\mathrm{ms}$ interval centered at the time of the maximal response. When neuronal activity at the time of maximal modulation statistically differed from that during the $50 \mathrm{~ms}$ interval centered on the stimulus presentation (one-tailed paired $t$ test, $p<0.01$ ), the neuronal modulation was defined as either facilitatory or suppressive; otherwise, the neuron was evaluated as no response. Neurons with early facilitatory and late suppressive responses were defined as Switch-type neurons. Those with only facilitatory responses during either or both trial phases were regarded as 
Reactive-type neurons. The remaining neurons with only suppressive responses were defined as Predictive-type neurons. Neurons that had no significant firing modulation during stimulus repetition but responded only to saccades were classified as Saccade-type neurons.

Properties of baseline firing. To characterize the basic firing patterns of neurons recorded from different nuclei in the thalamus, we also examined the baseline firing rate and its variability (Fano factor) in individual neurons. For each neuron, the baseline firing rate was measured during central fixation $1000 \mathrm{~ms}$ before the saccade target onset. Fano factor was measured as $\sigma^{2} / \mu$, where $\sigma$ and $\mu$ indicated the SD and mean of the spike count, respectively, in every nonoverlapping $100 \mathrm{~ms}$ during the $1000 \mathrm{~ms}$ fixation period before the target onset. These values were averaged for each location of electrode penetration $(0.5$ or $1.0 \mathrm{~mm}$ grid) and were filtered with two-dimensional Gaussians ( $\sigma 0.5$ $\mathrm{mm}, 2.5 \mathrm{~mm}$ square; Fig. $1 D$ ). When the distributions of the data for different types of neurons were compared, the values for each lateral coordinate of the recording site were averaged.

Histologic procedures. After completion of the experiments, one monkey was killed for histologic examination. The animal was deeply anesthetized with sodium pentobarbital $(>60 \mathrm{mg} / \mathrm{kg}$, i.p.) and transcardially perfused with PBS followed by $3.5 \%$ paraformaldehyde. The brain was then removed, blocked, and equilibrated with $30 \%$ sucrose. Coronal sections ( $100 \mu \mathrm{m}$ thickness) were cut on a freezing microtome and stained with cresyl violet.

\section{Results}

\section{Localization and classification of neurons}

We recorded from a total of 190 task-related thalamic neurons while monkeys attempted to detect a single omission of repetitive stimuli. Most neurons were located in the medial edge of the VL nucleus and the adjacent CL and MD nuclei of the thalamus (Fig. $1 B$ ). Among these neurons, 47 (25\%) were classified as Saccade-type neurons because they showed activity for saccades but did not exhibit firing modulation during stimulus repetition. The remaining 142 neurons (75\%) exhibiting a periodic activity during central fixation were further classified into three groups (Reactive-type, Predictive-type, and Switch-type neurons) according to the direction of firing modulation for each repetitive stimulus (see Materials and Methods). One exceptional neuron with early suppressive and late facilitatory responses was excluded from further analysis. Figure $1 C$ plots the recording sites of the four types of neurons relative to the midline and the anterior commissure (AC) in two monkeys. Most neurons were found between the $\mathrm{MD}$ and VL nuclei, and many were recorded from a few stereotaxic coordinates centered at $8 \mathrm{~mm}$ posterior to the $\mathrm{AC}$ and $4 \mathrm{~mm}$ lateral to the midline. The distribution of Predictive-type neurons differed from that of Reactive-type or Saccade-type neurons along the anteroposterior axis (two-sample Kolmogorov-Smirnov test, $p<0.05$ )
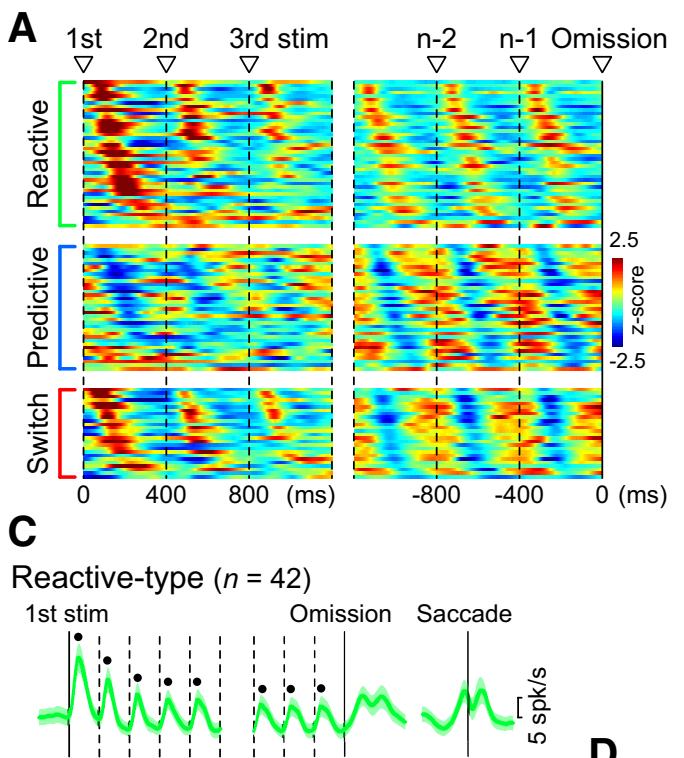

Predictive-type $(n=36)$

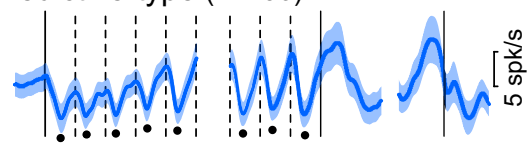

Switch-type $(n=26)$

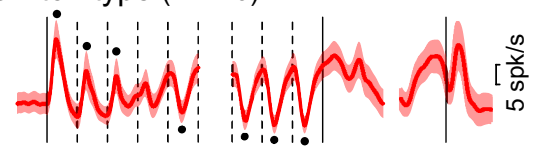

Saccade-type $(n=24)$
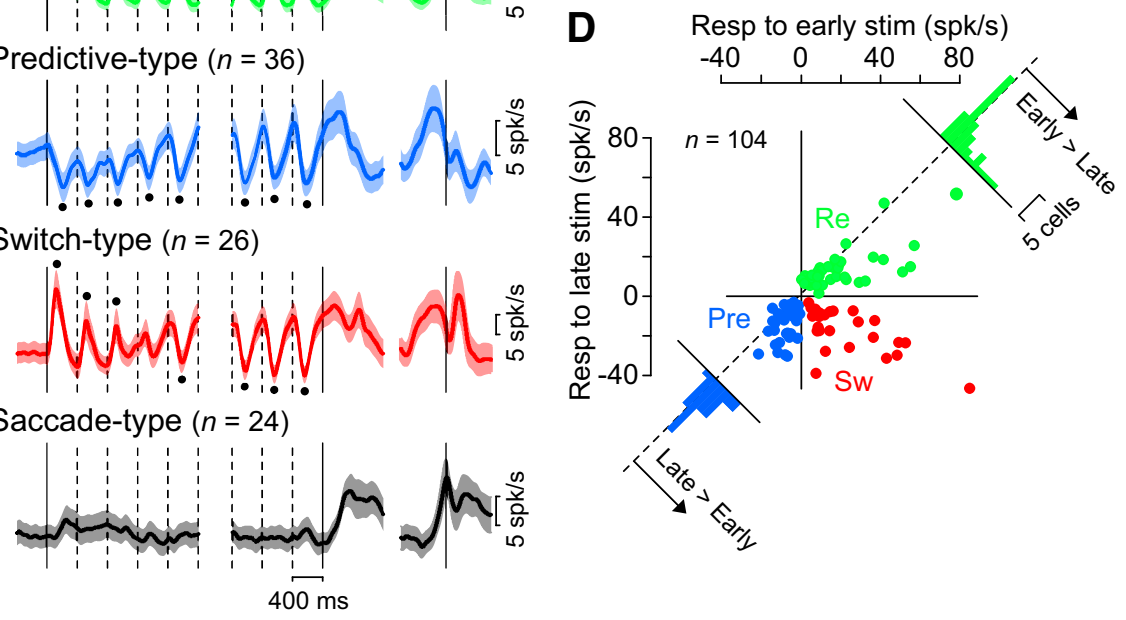

Figure 3. A, Activities of all recorded neurons with periodic firing modulation ( $n=42,36$, and 26 for Reactive-type, Predictive-type and Switch-type neurons, respectively). Each row represents single neuron and color indicates normalized firing rate (z-score) in trials with a $400 \mathrm{~ms}$ ISI. Data were normalized for each neuron. Neurons are sorted separately for the initial three ISIs (left) and the last three ISIs (right) to visualize the periodic firing modulation. Vertical dashed lines indicate stimulus (stim) timing. $\boldsymbol{B}$, Oriented black lines in each circle indicate the timing of peak activity in individual neurons. Each row plots the data from each type of neurons, while each column indicates the data measured for the early or late ISIs. The right-hand 0 indicates the timing of stimulus onset and the time during the $400 \mathrm{~ms} I S I$ passes in the counterclockwise direction (black arrow). A thick colored arrow indicates the angle of the summed vector for each panel. Note that the timing of peak activity for Reactive-type (green) and Predictive-type (blue) neurons remained consistent throughout the trial, but Switch-type neurons (red) showed a phase reversal. C, Time courses of population activity for different types of neurons. Shadow indicates the SEM. Black dot denotes the timing of maximal firing modulation (relative to the activity at stimulus onset) during each ISI. Note that Saccade-type neurons showed virtually no firing modulation during stimulus repetition but exhibited a transient response to saccades. $\boldsymbol{D}$, Comparison of the responses to the initial and the last three stimuli in the sequence. The direction and magnitude of the response measured relative to the activity at the time of stimulus onset. Green and blue histograms summarize the data for Reactive-type and Predictive-type neurons, respectively. spk, Spike.

but not along the mediolateral axis $(p>0.05)$, and Predictive-type neurons tended to be located more anteriorly (Wilcoxon rank-sum test, $p<0.01$ ). The distribution of Switch-type neurons did not differ from the other types of neurons along either axis (two-sample Kolmogorov-Smirnov test, $p>0.05$ ).

To better characterize neurons in different thalamic nuclei, we also examined the basic firing properties of individual neurons ( $n=267$, including 77 non-task-related neurons). Figure $1 D$ illustrates two-dimensional maps of baseline firing rate and Fano factor measured during central fixation before the onset of the saccade target (1000 ms; see Materials and Methods). Consistent with the previous studies (Glenn and Steriade, 1982; Steriade et 
al., 1993; Redinbaugh et al., 2020), neurons recorded in and lateral to the intralaminar thalamus (Fig. 1D, black dashed line) tended to exhibit higher and more variable baseline activity. This tendency was evident for all types of task-related neurons, and we did not find any significant difference between the neuron types (one-way ANOVA; firing rate: $F_{(3,185)}=1.61, p=0.19$; Fano factor: $\left.F_{(3,185)}=1.63, p=0.18\right)$.

Figure 2 illustrates representative examples of three types of neurons with periodic activity in trials with a $400 \mathrm{~ms}$ ISI. A Reactive-type neuron shown in Figure $2 \mathrm{~A}$ exhibited transient activity for each early stimulus in the sequence, but reduced activity during the later phase of the trial. This example neuron also elevated activity associated with saccades (Fig. 2A, right). Figure $2 B$ plots a Predictive-type neuron that showed no significant response during the initial few cycles but exhibited a suppressive activity for each stimulus as repetition progressed. The suppressive activity was followed by a slight rebound of activity, and consequently, the timing of peak activity was approximately aligned with the stimulus onset, similar to neurons previously reported in the cerebellar dentate nucleus (Ohmae et al., 2013; Uematsu et al., 2017). A Switch-type neuron (Fig. 2C) showed a transient activity for each early stimulus, but the timing of the peak gradually advanced and eventually coincided with the stimulus onset. Thus, the neuron reversed the direction of firing modulation for the stimulus, from early facilitation to late suppression, in each trial.

\section{Response properties of different types of neurons}

Further quantitative analyses were performed on 104 neurons with periodic activity that were examined in eight or more trials in each condition. Among them, 42 (40\%), 36 (35\%), and 26 (25\%) neurons were classified as Reactive-type, Predictive-type, and Switch-type neurons, respectively (Fig. $3 A, C$ ). For all types of neurons, the firing modulation before the stimulus omission did not differ significantly between the trials with ipsilateral and contralateral saccade targets (two-tailed paired $t$ test; Reactive type: $t_{(41)}=0.26, p=0.80$; Predictive type; $t_{(35)}=0.32, p=0.75$; Switch-type: $\left.t_{(25)}=1.16, p=0.26\right)$. Therefore, the data for both saccade directions were combined for further analyses of neuronal activity during fixation. In contrast, neuronal firing around the time of saccades $( \pm 150 \mathrm{~ms})$ showed significant directional modulation (Wilcoxon rank-sum test, $p<0.05$ ) for 17 Reactivetype (40\%), 13 Predictive-type (36\%), and 7 Switch-type (27\%) neurons. The proportions of these neurons were not significantly different across the three types of neurons $\left(\chi^{2}\right.$ test, $\left.p=0.52\right)$. More than half of these neurons showed a preference for contraversive saccades (57\%; 12, 5, and 4 neurons for Reactive-type, Predictive-type, and Switch-type neurons, respectively). In the population, the saccade-related activities were directional for Reactive-type and Switch-type neurons (two-tailed paired $t$ test; Reactive type: $t_{(41)}=-2.25, p=0.03$; Switch type, $t_{(25)}=-2.84$, $p<0.01)$, but not for Predictive-type neurons $\left(t_{(35)}=1.00\right.$, $p=0.32)$. Among 24 Saccade-type neurons that were examined for eight or more blocks, 5 (21\%) neurons showed a preference for ipsiversive saccades and $8(33 \%)$ for contraversive saccades (Wilcoxon rank-sum test, $p<0.05$ ). Saccade-type neurons overall did not show significant directional modulation in the population $\left(t_{(23)}=-1.14, p=0.26\right)$. Neurons with directional saccade modulation $(n=50)$ were found throughout the recording area, and its distribution did not differ from the rest of task-related neurons $(n=78)$ either along the mediolateral (KolmogorovSmirnov test, $p=0.073)$ or anteroposterior $(p=0.74)$ axis.
The time courses of individual neuronal activities in each group displayed properties similar to those in the examples shown in Figure 2. Reactive-type neurons increased their activity for each repetitive stimulus, and the peak consistently occurred after stimulus onset (Fig. $3 A$ ). In the population of Reactive-type neurons, the magnitude of the transient response gradually decreased (Fig. 3C, top, green traces). Conversely, Predictivetype neurons showed a suppressive response to each stimulus and the firing modulation gradually increased as the repetition progressed (Fig. 3C, top middle, blue traces). During the late part of the trial, the timing of peak activity was approximately aligned to the stimulus onset. Switch-type neurons exhibited a burst of activity for each of the initial few stimuli, but the activity gradually waned and instead a suppressive response to each stimulus developed. As a result, the peak activity initially lagged behind the stimulus onset but advanced gradually and finally occurred around the time of stimulus onset (Fig. $3 A, C$ ).

To assess the changes in the timing of neuronal activity during the stimulus repetition, Figure $3 B$ plots the peak timing relative to the stimulus onset measured from the means of neuronal activity during the initial (Fig. $3 B$, left panels) and the last (Fig. $3 B$, right) three ISIs for each neuron (Materials and Methods). In Figure 3, the angle of each black line indicates the phase within the $400 \mathrm{~ms}$ cycle, and 0 indicates the timing of stimulus onset. During the early part of the trial, the angles averaged $145^{\circ} \pm 19^{\circ}$ $\left(95 \% \mathrm{CI},-15^{\circ} \pm 26^{\circ}\right.$ to $\left.149^{\circ} \pm 27^{\circ}\right)$, which corresponded to $161 \pm 21,-17 \pm 28$, and $166 \pm 30 \mathrm{~ms}$ from the stimulus onset for Reactive-type, Predictive-type, and Switch-type neurons, respectively. During the late part of the trial, the values became $143^{\circ} \pm 21^{\circ}(159 \pm 23 \mathrm{~ms}), 3^{\circ} \pm 20^{\circ}(4 \pm 23 \mathrm{~ms})$, and $-12^{\circ} \pm 17^{\circ}$ $(-14 \pm 18 \mathrm{~ms})$, respectively. The angles for the early and late parts were statistically indistinguishable for both Reactive-type and Predictive-type neurons (Watson-Williams test; Reactive type: $F_{(1,82)}=0.03, p=0.86$; Predictive type: $F_{(1,70)}=1.44$, $p=0.23)$. Furthermore, the angles measured for the initial part of Switch-type neurons did not differ from those for Reactive-type neurons $\left(F_{(1,66)}=0.074, p=0.79\right)$, while the angles for the late part of Switch-type neurons did not differ from those for Predictive-type neurons $\left(F_{(1,60)}=1.48, p=0.23\right)$. Thus, Switchtype neurons showed properties similar to the other types of neurons depending on the trial phase.

To quantify the magnitude of response to each repetitive stimulus, the maximal firing modulation (difference in activity during $50 \mathrm{~ms}$ relative to that at the stimulus onset; Materials and Methods) was measured during the initial and the last three ISIs. Figure $3 D$ summarizes the data from individual neurons, with different colors indicating the different types of neurons. By definition, Switch-type neurons (red dots) showed facilitatory responses to the early stimuli and suppressive responses to the late stimuli. Reactive-type neurons (green) consistently exhibited facilitatory responses, but their magnitude was significantly greater for the early than the late stimuli in the sequence (Fig. $3 D$, green histogram; two-tailed paired $t$ test, $t_{(41)}=2.47$, $p<0.05$ ). In contrast, Predictive-type neurons (Fig. $3 D$, blue) always showed suppressive responses that were greater in magnitude for the late than early stimuli in the sequence $\left(t_{(35)}=4.54\right.$, $\left.p<10^{-4}\right)$.

In previous studies in our laboratory, neuronal modulation for each repetitive stimulus was proportional to the ISI in both the deep cerebellar nuclei (Ohmae et al., 2013) and the striatum (Kameda et al., 2019). We next examined the activities of three types of thalamic neurons for different ISIs. Figure $4 A$ compares the time courses of normalized population activity for different 
A
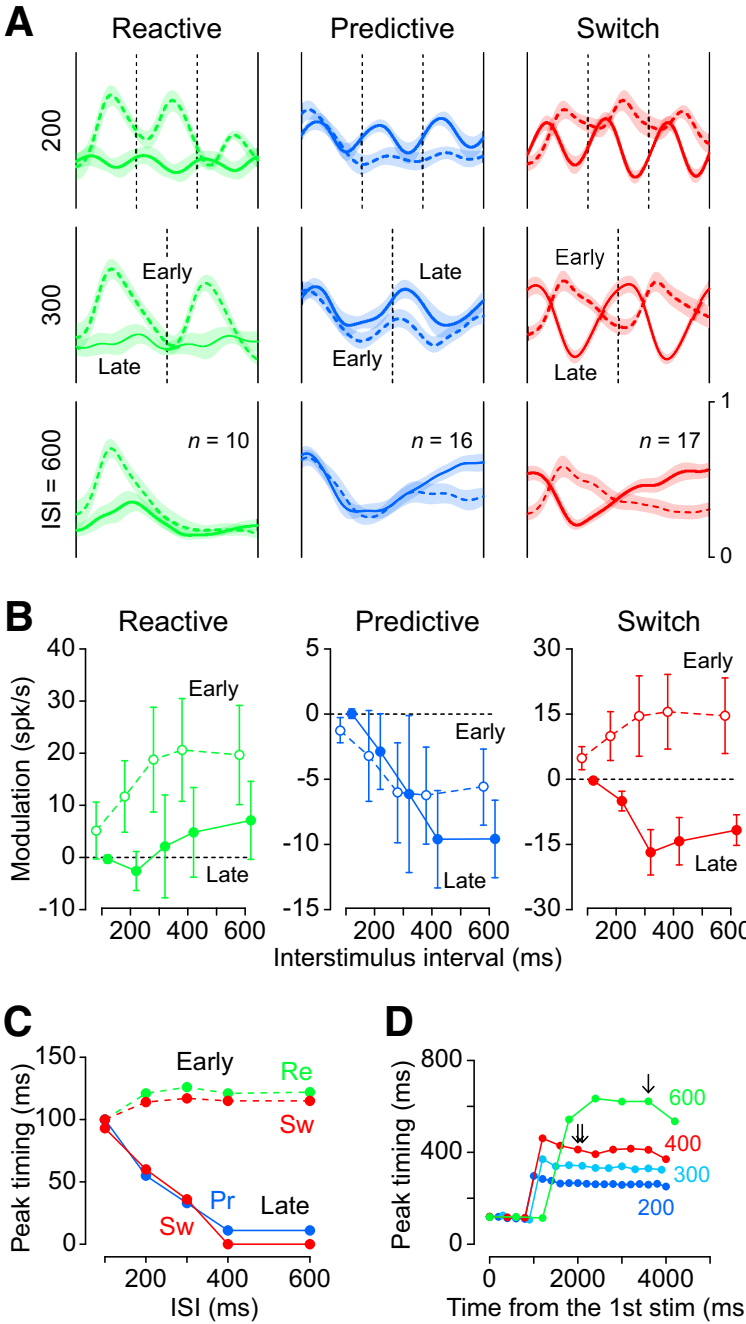

Predictive
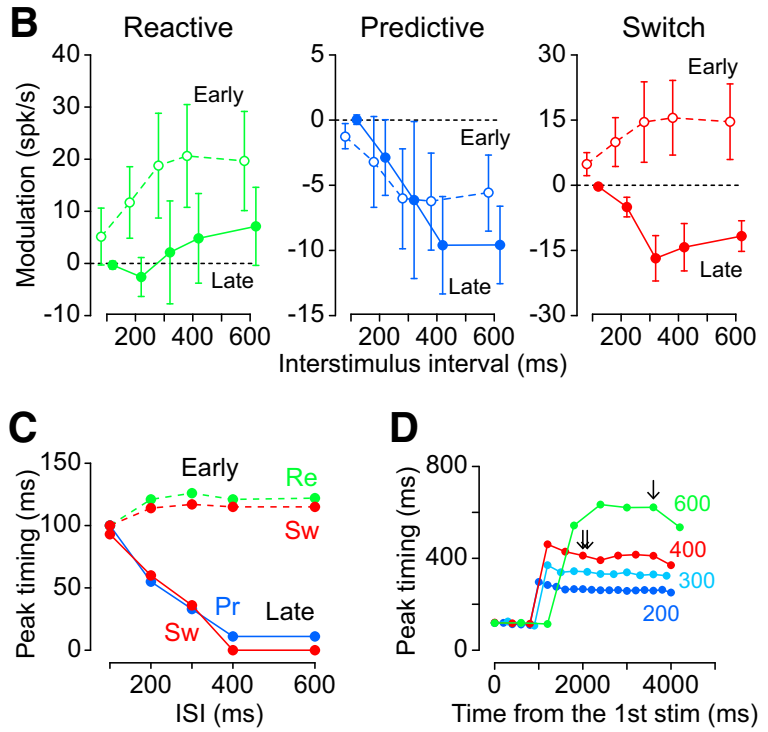

Figure 4. $\quad \boldsymbol{A}$, Time courses of normalized population activity for the early (dashed traces) and late (solid traces) stimuli (stim) in the sequence of different ISIs. Vertical lines indicate stimulus timing. Shadow indicates the SEM. $\boldsymbol{B}$, The response magnitudes of each type of neuron to the first three stimuli (open circles and dashed lines) and the last three stimuli (filled circles and solid lines) for different ISIs. Error bar indicates $95 \%$ confidence interval. Data points are slightly shifted horizontally for the presentation purpose only. C, Timing of peak activity for the early (data connected with dashed lines) and late (solid lines) stimuli in trials with different ISIs. For Reactive-type neurons, the peak timing was measured only for the early stimuli because there was virtually no firing modulation for the late stimuli, as in A. Similarly, the peak timing of Predictive-type neurons was measured only for the late stimuli. Note that the data for Switch-type neurons (red circles) during the early stimuli were similar to those for Reactive-type neurons (green), while those during the late stimuli were similar to Predictive-type neurons (blue). D, Timing of phase transition in Switch-type neurons for different ISIs. Timing of peak activity (measured during the interval starting from 100 ms after stimulus onset to $100 \mathrm{~ms}$ after the next stimulus) is plotted as a function of time from the first stimulus. Data were omitted unless half of the neurons were tested for eight or more trials. Downward arrows indicate the earliest timing of stimulus omission.

ISIs during the early (dashed lines) and late (solid lines) phases of the trial, which are aligned with the first stimulus and the stimulus omission, respectively. As seen previously (Fig. 3D), Reactive-type neurons exhibited greater activity for the early than late stimuli in the sequence, while Predictive-type neurons showed an opposite trend. Predictive-type neurons displayed greater firing modulation for longer ISIs during the late phase of the trial and showed a slight change in the timing of peak activity for different ISIs. Switch-type neurons showed periodic activity throughout the trial, and the time courses of activity during the early and late phases of the trial resembled those of Reactive-type and Predictive-type neurons, respectively. Switch-type neurons also exhibited an advancement of peak timing in each trial, while the amount of shift was only slight for shorter ISIs. Consequently, the timing of peak activity for shorter ISIs was delayed to the stimulus during the late part of the trial, while the peak timing for longer ISIs was approximately aligned with the stimulus onset.

Figure $4 B$ plots the magnitudes of response to the first and last three stimuli in the sequence for different ISIs. For Reactivetype neurons, the response to early stimuli (Fig. $4 B$, open circles connected with dashed lines) was consistently greater than that to late stimuli (Fig. $4 B$, filled circles), likely because of sensory adaptation. Although the firing modulation for the first stimulus was essentially the same across ISIs (Fig. $4 A$ ), the mean of the responses to the initial three stimuli was small for shorter ISIs. Predictive-type neurons showed greater firing modulation for longer stimulus intervals, similar to neurons in the cerebellum. Switch-type neurons reversed the direction of firing modulation from early facilitatory to late suppressive responses for all but the shortest (100 ms) ISIs.

Figure $4 C$ summarizes the timing of peak activity measured from the mean spike density profiles (the first and last three ISIs; Materials and Methods) for different ISIs. For both Reactive-type and Switch-type neurons, the timing of peak activity for early stimuli was $\sim 120 \mathrm{~ms}$ across all but $100 \mathrm{~ms}$ ISIs (data points connected with dashed lines). In contrast, for both Predictive-type and Switch-type neurons, the timing of peak activity during the late part of the trial (those connected with solid lines) gradually advanced as the ISI increased, and the peak activity occurred at the time of stimulus presentation for the ISI of 400 and $600 \mathrm{~ms}$. Thus, Switch-type neurons seemed to reflect the Reactive-type signals during the early phase of the trial and the Predictive-type signals during the late phase of the trial across different ISI conditions.

As seen in the time course of the population activity (Fig. 3C), the reversal in the direction of firing modulation in the Switchtype neurons occurred rather rapidly; the transition from facilitatory to suppressive responses occurred during the fourth ISI in the population of neurons. This was a sharp contrast from the gradual changes in response gain in Reactive-type and Predictive-type neurons (Fig. 3C). To examine the timing of phase transition during the trial, Figure $4 D$ plots the timing of peak activity during each ISI for Switch-type neurons as a function of time from the first stimulus in the sequence. For this analysis, the peak activity was searched for during the interval starting $100 \mathrm{~ms}$ after the stimulus onset, considering the neuronal response latency. The timing of peak activity suddenly changed well before the occurrence of the earliest stimulus omission $(3600 \mathrm{~ms}$ for $600 \mathrm{~ms}$ ISI, $2100 \mathrm{~ms}$ for $300 \mathrm{~ms}$ ISI, and $2000 \mathrm{~ms}$ for the other ISIs; downward arrows).

\section{Correlation with saccade latency}

To explore the possible causal role of signals in the central thalamus, we next examined the relationship between neuronal activity at the time of stimulus omission and behavioral latency. For this analysis, the data for each neuron were divided into six groups according to saccade latencies (with equal trial number), and the neuronal activity was compared among the groups. Figure $5 A$ plots the population activities for Switch-type neurons in trials with ipsilateral (Fig. $5 A$, left) or contralateral (Fig. $5 A$, right) saccade target. The data show that the neuronal activity just before the stimulus omission tended to be greater in trials with shorter saccade latencies, especially for the ipsilateral target 

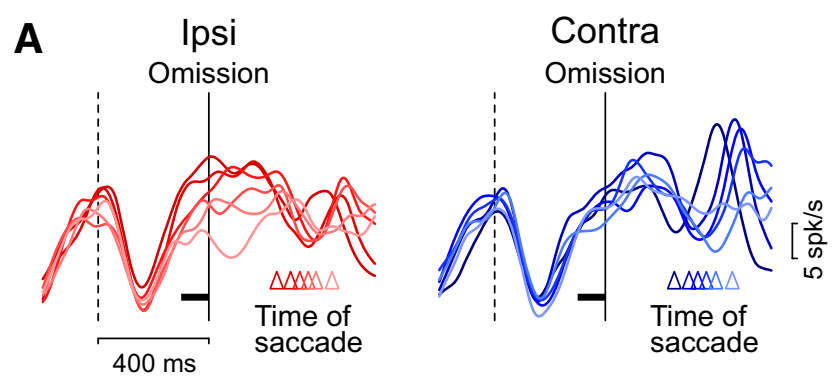

B

Ipsi
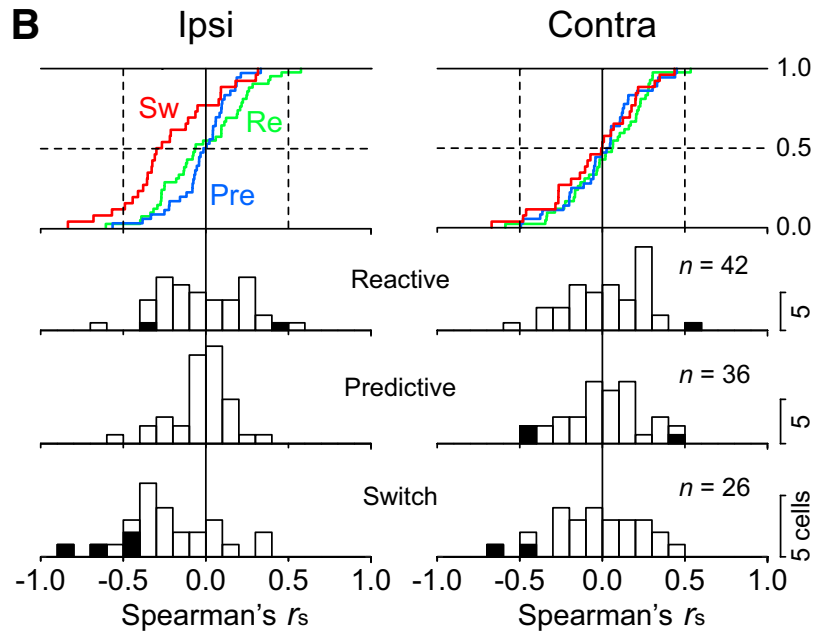

Figure 5. Correlation between neuronal activity before stimulus omission and saccade latency. $\boldsymbol{A}$, Time courses of the population activity of Switch-type neurons in ipsiversive (Ipsi; left) and contraversive (Contra; right) saccade trials with a 400 ms ISI. For each neuron and condition, the data were divided into six groups according to saccade latencies and the population activity was computed for each of the six groups. Triangle below the traces indicates the mean saccade latency for each group. Black bar under the traces indicates the $100 \mathrm{~ms}$ interval for measuring neuronal activity. $\boldsymbol{B}$, Cumulative distribution and histograms of Spearman's rank correlation coefficient for individual neurons. Different columns plot different saccade directions and colors in the cumulative plot indicate different types of neurons. Black bars in the standard histograms indicate neurons with a significant rank correlation $(p<0.05)$. Pre, Predictive; Re, Reactive; Sw, Switch.

(Fig. 5A). In the population of Switch-type neurons, the mean firing rates during $100 \mathrm{~ms}$ before the stimulus omission inversely correlated with the mean saccade latencies in ipsiversive saccade trials (Spearman's rank correlation coefficient $r_{\mathrm{s}}=-1.0$, $p<0.01)$, but not in contraversive saccade trials $\left(r_{\mathrm{s}}=-0.20\right.$, $p=0.71)$. No significant correlation was found for the populations of both Reactive-type and Predictive-type neurons for any saccade direction ( $r_{\mathrm{s}}$ range, -0.37 to $0.37 ; p>0.05$ ).

Correlation analysis was also performed for individual neurons. For each neuron, we calculated the Spearman's rank correlation coefficient between neuronal activity (spike count during $100 \mathrm{~ms}$ before stimulus omission) and saccade latency in each trial. Figure $5 B$ plots the cumulative distribution (top panel) and the standard histograms of the correlation coefficients for each type of neuron. Because the variation from trial to trial was large and the number of trial repeats was limited, only a few individual neurons showed a significant correlation $(p<0.05$; Fig. $5 B$, black bars). For Switch-type neurons (Fig. $5 B$, red trace, bottom histogram), the values for ipsiversive saccades were negative in most neurons (20 of 26 neurons, 77\%) and were significantly $<0$ in the population (two-tailed $t$ test: $t_{(25)}=-3.95, p<0.001$ ), while the correlation coefficients for contraversive saccades did not differ from $0\left(t_{(25)}=-0.70, p=0.49\right)$. The values for the other types of neurons also did not significantly differ from 0 for either saccade direction in trials with a 400 ms ISI (Reactive-type: ipsiversive saccade, $t_{(41)}=-0.35, p=0.72$; contraversive saccade, $t_{(41)}=0.86, p=0.39$; Predictive-type: ipsiversive saccade, $t_{(35)}=$ $-0.74, p=0.46$; contraversive saccade, $\left.t_{(35)}=0.06, p=0.95\right)$. The correlation coefficients for Switch-type neurons differed from those for the other types of neurons in trials with ipsiversive saccades (one-way ANOVA, $F_{(2,101)}=6.70, p<0.01$; multiple comparisons with Tukey's method, $p<0.05)$ but not in trials with contraversive saccades $\left(F_{(2,101)}=0.64, p=0.53\right)$. The correlation coefficients of Switch-type neurons in trials with a $600 \mathrm{~ms}$ ISI were also significantly $<0$ for both saccade directions (two-tailed $t$ test; ipsiversive: $t_{(16)}=-2.3, p<0.05$; contraversive: $t_{(16)}=$ $-4.2, p<0.01$ ), while those for the other types of neurons were not. In trials with shorter ISIs (100-300 ms), the correlation coefficients did not differ from 0 in the population, except for Reactive-type neurons in the condition of contralateral saccades with a $300 \mathrm{~ms}$ ISI (two-tailed $t$ test; $t_{(9)}=-2.8, p<0.05$ ). Finally, when the data for all types of neurons were combined, the correlation coefficients differed from 0 for both saccade directions in the $600 \mathrm{~ms}$ ISI condition $(p<0.01)$, and for ipsiversive and contraversive saccades in the 400 and $300 \mathrm{~ms}$ ISI conditions, respectively.

\section{Alteration of neuronal activity in the absence of temporal prediction}

Our behavioral paradigm required motor preparation along with the prediction of the next stimulus timing. To examine how these task requirements affected neuronal activity, we devised two variants of the behavioral task. First, we used the nontarget missing oddball task (Fig. 6A) to eliminate both motor and prediction components. In this task, the audiovisual stimulus was presented repeatedly, as in the original task, but the saccade target never appeared. The animals were only required to maintain fixation throughout the trial to obtain a reward. Because the animals did not need to respond to the stimulus omission, they had to neither predict stimulus timing nor prepare for saccades. Our previous study showed that neurons in the cerebellar dentate nucleus reduced their firing modulation in this task (Ohmae et al., 2013).

Figure $6 B$ compares the population activities between the nontarget and randomly presented original tasks for the three types of thalamic neurons. Qualitatively, Predictive-type and Switch-type neurons reduced the magnitude of periodic firing modulation, but Reactive-type neurons only exhibited a slight change in baseline activity. When the magnitude of responses during the three ISIs just before the stimulus omission were compared between the conditions, Predictive-type and Switchtype neurons significantly reduced the activity in the nontarget trials (two-tailed paired $t$ test; Predictive-type: $t_{(27)}=-4.75$ $p<10^{-4}$; Switch-type: $t_{(18)}=-4.27, p<10^{-3}$; Fig. $6 C$ ), while Reactive-type neurons did not $\left(t_{(29)}=0.17, p=0.87\right.$; data not shown).

Second, to distinguish neuronal activities related to motor preparation from those related to the prediction of stimulus timing, we introduced the explicit deviant task in which monkeys generated a saccade but did not need to predict the next stimulus timing (Fig. 7A). In this task, a green rather than a red fixation point was presented, and one repetitive stimulus in the series was replaced with a red square $\left(2^{\circ}\right)$ and a low-frequency tone $(677 \mathrm{~Hz}$, deviant oddball). To receive a reward, monkeys were required to make an immediate saccade in response to the deviant stimulus. In this case, the animals only needed to attend the stimulus color during the preparation of a saccade. 


\section{A Non-target task}
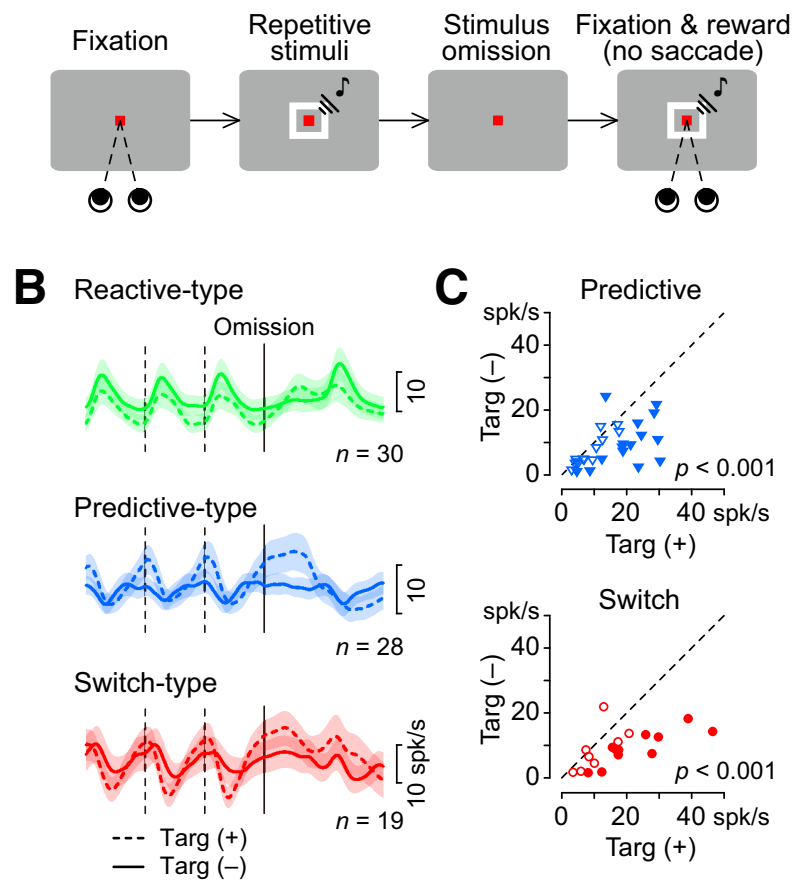

Figure 6. Context-dependent changes in neuronal activity in the nontarget task. $A$, In this task, the basic configuration was same as the missing oddball trials (Fig. 1A) but the saccade target (Targ) never appeared. Animals were required to maintain fixation for $>1200 \mathrm{~ms}$ following the stimulus omission to obtain a reward. $\boldsymbol{B}$, Time courses of the population activity for different types of neurons. Note that the firing modulation for each stimulus in the nontarget trials was reduced for Predictive-type and Switch-type neurons, but remained unchanged for Reactive-type neurons. Shadow indicates the SEM. C, Comparisons of the magnitude of firing modulation between trials with and without saccade target in individual neurons. Filled symbols indicate neurons exhibiting significant difference (unpaired $t$ test, $p<0.05)$. spk, Spike.

Compared with the activity during the original missing oddball trials, both the representative and the population of Switchtype neurons exhibited reduced activity in the explicit deviant trials, especially during the late part of the trial (Fig. $7 B, C$ ). Furthermore, the timing of peak activity was slightly delayed from the stimulus onset throughout the deviant oddball trials (Fig. $7 B, C$, red traces), while the timing of peak activity gradually advanced during the original missing oddball trials (Fig. $7 B, C$, blue). To quantify this, we searched for the peak in activity in the means of spike density profiles for the first and the last three ISIs (Fig. 4C). The timing of peak activity for the early stimuli was comparable between the missing and deviant conditions (94 vs $86 \mathrm{~ms}$ ), but the timing for the late stimuli was delayed in the deviant trials ( 0 and $54 \mathrm{~ms}$ for the missing and deviant conditions, respectively). These results indicated that the advanced activity before stimulus presentation in Switch-type neurons (Fig. $4 B, C)$ reflected the prediction of the next stimulus timing. Because only seven neurons were tested, the neuronal activity just before $(100 \mathrm{~ms})$ and shortly after (50-200 ms) the oddball did not differ significantly between the two stimulus conditions (two-tailed paired $t$ test; $t_{(6)}=-1.5, p=0.18$, and $t_{(6)}=-0.43, p$ $=0.68$, respectively), although the activity tended to be greater for the missing than for the deviant conditions (Fig. 7C).

To examine whether thalamic neurons carried the prediction error signals, we next compared the firing rate just after the stimulus omission with that after the previous stimulus. The activity following the stimulus omission (50-200 ms) was greater than
A Deviant oddball task
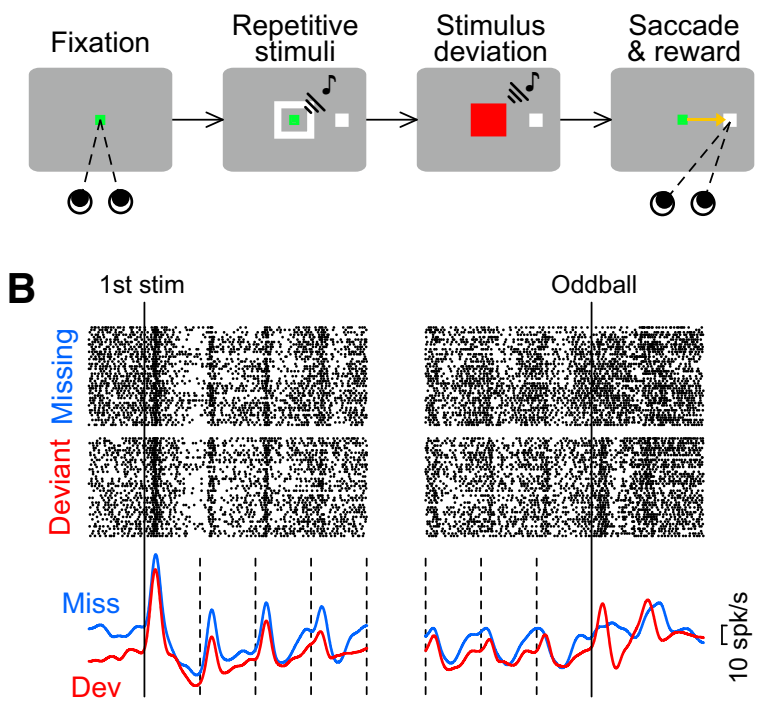

\section{Population activity $(n=7)$}
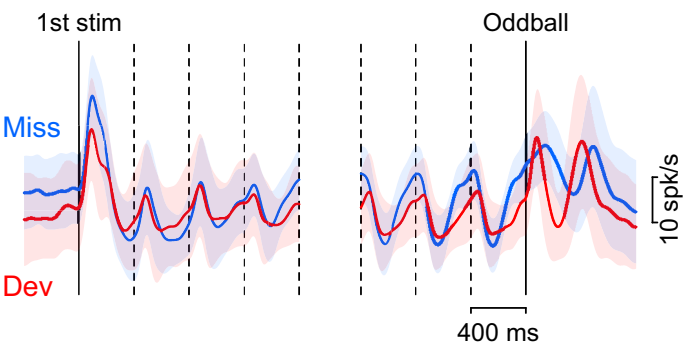

Figure 7. Context-dependent changes in neuronal activity in the explicit deviant (Dev) task. $\boldsymbol{A}$, In this task, a green rather than a red fixation point was presented to identify the trial type. Monkeys were required to make an immediate saccade in response to the deviation in color and frequency of the repetitive stimulus (stim). B, Activity of a Switch-type neuron. Note that both the magnitude of firing modulation and the timing of peak activity altered in the explicit deviant trials (red trace) compared with the original missing (Miss) oddball trials (blue). C, Population activity of Switch-type neurons. Shadow indicates the SEM.

that after the regular stimulus in Predictive-type and Switch-type neurons (two-tailed paired $t$ test; Predictive type: $t_{(35)}=-5.6$, $p<0.01$; Switch type: $\left.t_{(25)}=-6.3, p<0.01\right)$, but was not in Reactive-type neurons $\left(t_{(41)}=1.5, p=0.14\right)$. Furthermore, when comparing the responses to stimulus omission between the nontarget trials and the omission detection trials, Predictive-type and Switch-type neurons showed greater activity in the omission detection task (two-tailed paired $t$ test; Predictive type: $t_{(27)}=$ $-2.3, p<0.05$; Switch type: $\left.t_{(18)}=-2.2, p<0.05\right)$, while the Reactive-type neurons did not show a significant difference (two-tailed paired $t$ test; $t_{(29)}=0.54, p=0.59$ ). Thus, for both Predictive-type and Switch-type neurons, the activity just after the stimulus omission was greater when the animals were required to actively detect stimulus omission. Although the difference in activity was largely attributed to the lack of suppressive response to each repetitive stimulus, these results indicated that some thalamic neurons might represent prediction error for the absence of regular stimulus.

\section{Discussion}

The present study revealed a part of the neural mechanism of temporal prediction in the primate central thalamus. We found 
that thalamic neurons exhibited periodic activity when the animals attempted to detect a single omission of isochronous repetitive stimuli. Individual neurons responded differently to the stimulus at the beginning and end of the sequence and were classified into three groups according to the direction of firing modulation for each stimulus. The thalamus appears to modify and transmit signals, rather than simply relaying a faithful copy of subcortical signals to the cerebral cortex.

\section{Properties and generating mechanism of neuronal signals in the central thalamus}

Reactive-type neurons exhibited a transient activity for each stimulus, which was gradually attenuated as the repetition progressed (Figs. 2A, 3C). The decrement of sensory gain for repeated stimulus exposure resembled sensory adaptation, which is a ubiquitous finding in the sensory system (Dean et al., 2008; Netser et al., 2011). Since the central thalamus receives inputs from the cortical and subcortical visual areas including the posterior parietal cortex (Taktakishvili et al., 2002) and the superior colliculus (SC; Benevento and Fallon, 1975), Reactive-type neurons might reflect the properties of these sensory neurons. Previous studies have shown that the amount of sensory adaptation in visual responsive neurons in the frontal eye field and the SC is proportional to the ISI (Mayo and Sommer, 2008), providing a possible neural code for time perception (Mayo and Sommer, 2013). However, in our experimental condition, the firing modulation of Reactive-type neurons at the time of stimulus omission was only small or even absent, and, by definition, they showed reactive rather than predictive responses (Figs. 3C, $4 B$ ). Therefore, the Reactive-type signal is unlikely to be useful in predicting stimulus timing and detecting unexpected stimulus omission in our experimental paradigm.

Conversely, Predictive-type neurons gradually increased the magnitude of periodic firing modulation, that is, in the direction opposite to sensory adaptation (Figs. $2 B, 3 C$ ). The time course of Predictive-type neuronal activity was similar to that reported previously in the cerebellar dentate nucleus (Ohmae et al., 2013), suggesting that Predictive-type signals might come from the cerebellum. Indeed, the neuronal modulation in both structures was proportional to the ISI (Fig. $4 B$ ), and the activity greatly decreased as the animals passively viewed the repetitive stimulus in the nontarget condition (Fig. 6C; Ohmae et al., 2013).

We also found a novel type of neuron in the central thalamus. Switch-type neurons exhibited a transient response to each of the initial few stimuli in the sequence, but, after several cycles, the direction of firing modulation reversed, and the activity decreased for each repetitive stimulus (Figs. 2C, 3C). The changes in the timing of peak activity occurred rather abruptly and preceded the earliest stimulus omission in trials with all but $100 \mathrm{~ms}$ ISIs (Fig. 4D). Switch-type neurons decreased the firing modulation in trials without a saccade target, where neither temporal prediction nor saccade preparation were necessary (Fig. 6C). Furthermore, the activity predicting the stimulus onset was also reduced in the explicit deviant condition that required saccade generation without a need for temporal prediction (Fig. 7). These results suggest that the Switch-type activity before stimulus presentation might reflect the temporal prediction of sensory events rather than the preparation of saccades. Thus, individual Switch-type neurons appeared to represent the transition from a reactive to a predictive code for stimulus timing. Because only Switch-type neurons showed a significant correlation with saccade latency (Fig. $5 B$ ), this class
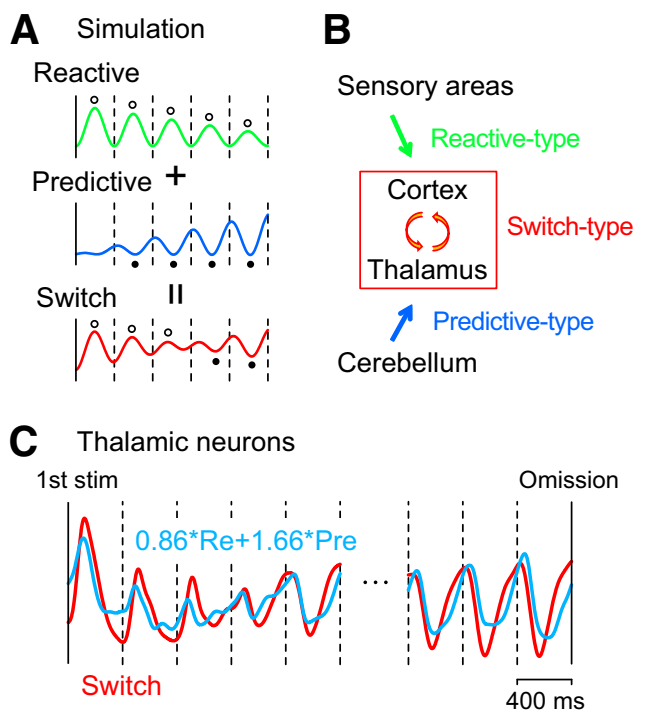

Figure 8. Integration of Reactive-type (Re) and Predictive-type (Pre) signals might account for the time course of Switch-type signals. $\boldsymbol{A}$, Simple summation of sine and cosine waves with gradual amplitude modulation can generate phase transition. $\boldsymbol{B}$, Hypothetical diagram generating the Switch-type signals. Thalamocortical network receives Reactive-type signals from sensory areas (green arrow) and Predictive-type signals from the cerebellum (blue). These signals are integrated in the thalamocortical network to generate Switch-type signals. C, Red trace indicates the data for Switch-type neurons (replotted from Fig. 3C). Blue trace indicates the weighted sum of the activities of Reactive-type and the Predictive-type neurons $(0.86 * \operatorname{Re}+1.66 *$ Pre + bias). The optimal weights and biases were computed using the least squares $\left(r^{2}=0.58\right)$.

of neurons might carry more advanced signals in the system that detects stimulus omission.

Based on the time courses of neuronal activity, we speculated that the Switch-type signal could be generated by integrating the other two types of signals. For example, the summation of a sine wave of gradually decreasing amplitude and a cosine wave of increasing amplitude can successfully reproduce a phase transition (Fig. $8 A$ ). To test this possibility, we attempted to generate a Switch-type signal from the population activities of Reactive-type and Predictive-type neurons. When considering neuronal activity during the initial five and the last three ISIs, a linear summation of the Reactive-type and the Predictive-type signals with optimal weights (least squares) could explain 58\% variation of the Switch-type signal (Fig. 8C).

Several features of the Switch-type signal also supported the hypothesis of integration. Similar to Reactive-type neurons, the transient response to the initial few stimuli in the sequence increased as a function of the ISI (Fig. $4 B$ ), while the timing of the peak remained unchanged (Fig. 4C). Conversely, both Switch-type and Predictive-type neurons showed suppressive responses to the later stimuli in the sequence, and the magnitude of modulation for each stimulus was also proportional to ISI (Fig. $4 B$ ). In both populations, the timing of peak activity after many cycles gradually advanced as the ISI lengthened, and was aligned with stimulus onset for the 400 and $600 \mathrm{~ms}$ ISIs (Fig. $4 C)$. Furthermore, both Switch-type and Predictive-type neurons attenuated the periodic response in the nontarget trials (Fig. 6C). In the present study, we were unable to determine the site of integration of the two different signals, but because our previous exploration in the cerebellum and the striatum failed to find the Switch-type signals, this integration might occur within the thalamocortical network (Fig. 8B). 
One might argue that our classification of neurons was arbitrary, and that the three types of neurons could be a continuum. Although Predictive-type neurons appear to be distinct from the other types of neurons, the difference between Switch-type and Reactive-type neurons might depend on the number of Predictivetype signals to be integrated. For example, the attenuated response in Reactive-type neurons could be generated by integrating the Predictive-type signals that gradually increased the magnitude of suppressive response. In relation to this, a recent study has demonstrated that the balance of excitatory and inhibitory signals in mutual inhibitory circuitry can explain many features of temporal prediction for rhythmic events (Egger et al., 2020). The three types of neurons in the thalamus might reflect such interactions.

\section{Possible roles of signals in the central thalamus for temporal prediction}

The central thalamus mutually connects with cortical areas relevant to temporal information processing, such as the dorsomedial frontal cortex (Merchant et al., 2011), the dorsolateral prefrontal cortex (Oshio et al., 2006), and the posterior parietal cortex (Schneider and Ghose, 2012; Jazayeri and Shadlen, 2015). Both Predictive-type and Switch-type neurons in the thalamus may provide internal prediction signals of stimulus timing to these cortical areas. These signals might be integrated with sensory signals in the cortical network to generate the prediction error signals for stimulus absence, which may directly trigger movements during the missing oddball task. The signals from the thalamus may also contribute to regulating the time course of prediction signals in the cortex (Egger et al., 2019) or in the striatum through the direct projections through the CL nucleus (Ichinohe et al., 2000; Hoshi et al., 2005).

In our experimental paradigm, the monkeys needed to learn the stimulus tempo in every trial because the ISI varied from trial to trial. During the initial phase of each trial, the animals would detect the stimulus just after its onset, but after several cycles, they became possible to accurately predict the next stimulus timing. Thus, the internal representation of stimulus timing may exhibit a phase transition, as seen in the time course of Switch-type neuronal activity. The transition from reactive to predictive code for stimulus timing resembles a gradual change in reaction time when attempting to perform synchronized movements with a regular rhythm (Takeya et al., 2017; Gámez et al., 2018). Like the present perceptual task, a similar Switch-type signal could also be observed during tasks requiring synchronized movements.

The present results suggest that the central thalamus may play a role in integrating signals derived from multiple sources. Predictive-type neurons likely transmit signals from the cerebellum, while Switch-type neurons showed properties of both the sensory and temporal prediction signals. It has been proposed that the thalamus not only relays a copy of signals but also actively integrates information from multiple areas. For example, in the sensory system, the thalamus is known to integrate the ascending subcortical sensory information with the descending driver signal originating from the cortex to perform supralinear summation (Groh et al., 2014; Sherman, 2016). In the motor system, the thalamus receives inputs from the motor cortical areas along with the subcortical signals arising from the cerebellum and the basal ganglia, possibly playing a role in boosting motor commands (Kakei et al., 2001; Kunimatsu and Tanaka, 2010; Bosch-Bouju et al., 2013). Furthermore, a recent study in rodents has shown that the maintenance of preparatory activity in the motor thalamus requires direct inputs from the premotor cortex (Guo et al., 2017). Although these previous findings prompted us to infer that Switch-type signals may be generated within the thalamocortical network, it is also possible that these signals are generated in subcortical structures that have never been explored during the present task. These signals may be discovered in future recording studies in the cerebral cortex.

\section{References}

Ahissar E, Oram T (2015) Thalamic relay or cortico-thalamic processing? Old question, new answers. Cereb Cortex 25:845-848.

Alexander GE, DeLong MR, Strick PL (1986) Parallel organization of functionally segregated circuits linking basal ganglia and cortex. Annu Rev Neurosci 9:357-381.

Allman MJ, Meck WH (2012) Pathophysiological distortions in time perception and timed performance. Brain 135:656-677.

Benevento LA, Fallon JH (1975) The ascending projections of the superior colliculus in the rhesus monkey (Macaca mulatta). J Comp Neurol 160:339-361.

Berens P (2009) CircStat: a MATLAB toolbox for circular statistics. J Stat Softw 31:1-21.

Bosch-Bouju C, Hyland BI, Parr-Brownlie LC (2013) Motor thalamus integration of cortical, cerebellar and basal ganglia information: implications for normal and parkinsonian conditions. Front Comput Neurosci 7:163.

Bostan AC, Strick PL (2018) The basal ganglia and the cerebellum: nodes in an integrated network. Nat Rev Neurosci 19:338-350.

Costello MG, Zhu DT, May PJ, Salinas E, Stanford TR (2016) Task dependence of decision- and choice-related activity in monkey oculomotor thalamus. J Neurophysiol 115:581-601.

Dean I, Robinson BL, Harper NS, McAlpine D (2008) Rapid neural adaptation to sound level statistics. J Neurosci 28:6430-6438.

Egger SW, Remington ED, Chang CJ, Jazayeri M (2019) Internal models of sensorimotor integration regulate cortical dynamics. Nat Neurosci 22:1871-1882.

Egger SW, Le NM, Jazayeri M (2020) A neural circuit model for human sensorimotor timing. Nat Commun 11:3933.

Fujioka T, Trainor LJ, Large EW, Ross B (2012) Internalized timing of isochronous sounds is represented in neuromagnetic $\beta$ oscillations. J Neurosci 32:1791-1802.

Gámez J, Yc K, Ayala YA, Dotov D, Prado L, Merchant H (2018) Predictive rhythmic tapping to isochronous and tempo changing metronomes in the nonhuman primate. Ann N Y Acad Sci 1423:396-414.

Glenn LL, Steriade M (1982) Discharge rate and excitability of cortically projecting intralaminar thalamic neurons during waking and sleep states. J Neurosci 2:1387-1404.

Groh A, Bokor H, Mease RA, Plattner VM, Hangya B, Stroh A, Deschenes M, Acsády L (2014) Convergence of cortical and sensory driver inputs on single thalamocortical cells. Cereb Cortex 24:3167-3179.

Guo ZV, Inagaki HK, Daie K, Druckmann S, Gerfen CR, Svoboda K (2017) Maintenance of persistent activity in a frontal thalamocortical loop. Nature 545:181-186.

Haber SN, Calzavara R (2009) The cortico-basal ganglia integrative network: the role of the thalamus. Brain Res Bull 78:69-74.

Harrington DL, Castillo GN, Fong CH, Reed JD (2011) Neural underpinnings of distortions in the experience of time across senses. Front Integr Neurosci 5:32.

Hoshi E, Tremblay L, Féger J, Carras PL, Strick PL (2005) The cerebellum communicates with the basal ganglia. Nat Neurosci 8:1491-1493.

Ichinohe N, Mori F, Shoumura K (2000) A di-synaptic projection from the lateral cerebellar nucleus to the laterodorsal part of the striatum via the central lateral nucleus of the thalamus in the rat. Brain Res 880:191-197.

Jazayeri M, Shadlen MN (2015) A neural mechanism for sensing and reproducing a time interval. Curr Biol 25:2599-2609.

Kakei S, Na J, Shinoda Y (2001) Thalamic terminal morphology and distribution of single corticothalamic axons originating from layers 5 and 6 of the cat motor cortex. J Comp Neurol 437:170-185.

Kameda M, Ohmae S, Tanaka M (2019) Entrained neuronal activity to periodic visual stimuli in the primate striatum compared with the cerebellum. Elife 8:e48702

Kato S, Fukabori R, Nishizawa K, Okada K, Yoshioka N, Sugawara M, Maejima Y, Shimomura K, Okamoto M, Eifuku S, Kobayashi K (2018) Action selection and flexible switching controlled by the intralaminar thalamic neurons. Cell Rep 22:2370-2382. 
Kunimatsu J, Tanaka M (2010) Roles of the primate motor thalamus in the generation of antisaccades. J Neurosci 30:5108-5117.

Mair RG, Hembrook JR (2008) Memory enhancement with event-related stimulation of the rostral intralaminar thalamic nuclei. J Neurosci 28:14293-14300.

Mayo JP, Sommer MA (2008) Neuronal adaptation caused by sequential visual stimulation in the frontal eye field. J Neurophysiol 100:1923-1935.

Mayo JP, Sommer MA (2013) Neuronal correlates of visual time perception at brief timescales. Proc Natl Acad Sci U S A 110:1506-1511.

McFarland NR, Haber SN (2002) Thalamic relay nuclei of the basal ganglia form both reciprocal and nonreciprocal cortical connections, linking multiple frontal cortical areas. J Neurosci 22:8117-8132.

Merchant H, Zarco W, Pérez O, Prado L, Bartolo R (2011) Measuring time with different neural chronometers during a synchronization-continuation task. Proc Natl Acad Sci U S A 108:19784-19789.

Merchant H, Harrington DL, Meck WH (2013) Neural basis of the perception and estimation of time. Annu Rev Neurosci 36:313-336.

Mitchell AS, Dalrymple-Alford JC (2006) Lateral and anterior thalamic lesions impair independent memory systems. Learn Mem 13:388-396.

Netser S, Zahar Y, Gutfreund Y (2011) Stimulus-specific adaptation: can it be a neural correlate of behavioral habituation? J Neurosci 31:17811-17820.

Newman LA, Mair RG (2007) Cholinergic modulation of visuospatial responding in central thalamus. Eur J Neurosci 26:3543-3552.

Ohmae S, Uematsu A, Tanaka M (2013) Temporally specific sensory signals for the detection of stimulus omission in the primate deep cerebellar nuclei. J Neurosci 33:15432-15441.

Oshio K, Chiba A, Inase M (2006) Delay period activity of monkey prefrontal neurones during duration-discrimination task. Eur J Neurosci 23:27792790.

Parent A, Hazrati LN (1995) Functional anatomy of the basal ganglia. I. The cortico-basal ganglia-thalamo-cortical loop. Brain Res Brain Res Rev 20:91-127.

Paton JJ, Buonomano DV (2018) The neural basis of timing: distributed mechanisms for diverse functions. Neuron 98:687-705

Prevosto V, Sommer MA (2013) Cognitive control of movement via the cerebellar-recipient thalamus. Front Syst Neurosci 7:56.

Rao SM, Harrington DL, Haaland KY, Bobholz JA, Cox RW, Binder JR (1997) Distributed neural systems underlying the timing of movements. J Neurosci 17:5528-5535.

Redinbaugh MJ, Phillips JM, Kambi NA, Mohanta S, Andryk S, Dooley GL, Afrasiabi M, Raz A, Saalmann YB (2020) Thalamus modulates consciousness via layer-specific control of cortex. Neuron 106:66-75.e12.

Rovó Z, Ulbert I, Acsády L (2012) Drivers of the primate thalamus. J Neurosci 32:17894-17908.

Saalmann YB (2014) Intralaminar and medial thalamic influence on cortical synchrony, information transmission and cognition. Front Syst Neurosci 8:83.

Schiff ND (2008) Central thalamic contributions to arousal regulation and neurological disorders of consciousness. Ann N Y Acad Sci 1129:105118
Schlag J, Schlag-Rey M (2002) Through the eye, slowly: delays and localization errors in the visual system. Nat Rev Neurosci 3:191-215.

Schlag-Rey M, Schlag J (1989) The central thalamus. Rev Oculomot Res 3:361-390.

Schneider BA, Ghose GM (2012) Temporal production signals in parietal cortex. PLoS Biol 10:e1001413.

Sherman SM (2016) Thalamus plays a central role in ongoing cortical functioning. Nat Neurosci 19:533-541.

Steriade M, Curró Dossi R, Contreras D (1993) Electrophysiological properties of intralaminar thalamocortical cells discharging rhythmic (approximately $40 \mathrm{~Hz}$ ) spike-bursts at approximately $1000 \mathrm{~Hz}$ during waking and rapid eye movement sleep. Neuroscience 56:1-9.

Takeya R, Kameda M, Patel AD, Tanaka M (2017) Predictive and tempoflexible synchronization to a visual metronome in monkeys. Sci Rep 7:6127.

Taktakishvili O, Sivan-Loukianova E, Kultas-Ilinsky K, Ilinsky IA (2002) Posterior parietal cortex projections to the ventral lateral and some association thalamic nuclei in Macaca mulatta. Brain Res Bull 59:135-150.

Tanaka M (2005) Involvement of the central thalamus in the control of smooth pursuit eye movements. J Neurosci 25:5866-5876.

Tanaka M (2006) Inactivation of the central thalamus delays self-timed saccades. Nat Neurosci 9:20-22.

Tanaka M (2007a) Cognitive signals in the primate motor thalamus predict saccade timing. J Neurosci 27:12109-12118.

Tanaka M (2007b) Spatiotemporal properties of eye position signals in the primate central thalamus. Cereb Cortex 17:1504-1515.

Tanaka M, Kunimatsu J (2011) Contribution of the central thalamus to the generation of volitional saccades. Eur J Neurosci 33:2046-2057.

Uematsu A, Ohmae S, Tanaka M (2017) Facilitation of temporal prediction by electrical stimulation to the primate cerebellar nuclei. Neuroscience 346:190-196.

Van der Werf YD, Witter MP, Groenewegen HJ (2002) The intralaminar and midline nuclei of the thalamus. Anatomical and functional evidence for participation in processes of arousal and awareness. Brain Res Brain Res Rev 39:107-140.

van Donkelaar P, Stein JF, Passingham RE, Miall RC (2000) Temporary inactivation in the primate motor thalamus during visually triggered and internally generated limb movements. J Neurophysiol 83:2780-2790.

Wang J, Narain D, Hosseini EA, Jazayeri M (2018) Flexible timing by temporal scaling of cortical responses. Nat Neurosci 21:102-110.

Wyder MT, Massoglia DP, Stanford TR (2003) Quantitative assessment of the timing and tuning of visual-related, saccade-related, and delay period activity in primate central thalamus. J Neurophysiol 90:2029-2052.

Wyder MT, Massoglia DP, Stanford TR (2004) Contextual modulation of central thalamic delay-period activity: representation of visual and saccadic goals. J Neurophysiol 91:2628-2648.

Yamawaki N, Shepherd GM (2015) Synaptic circuit organization of motor corticothalamic neurons. J Neurosci 35:2293-2307. 